\title{
Performance of Space-Time Block Coding in Powerline and Satellite Communications
}

\author{
Anna Papaioannou, Student Member, IEEE, George D. Papadopoulos, Student Member, IEEE, and Fotini-Niovi \\ Pavlidou, Senior Member, IEEE
}

\begin{abstract}
In this paper Space-Time Block Coding (STBC) is examined in powerline and satellite communications, comparing the gains that this coding-modulation technique provides to these communications systems. The powerline environment is assumed to be a frequency-selective, multipath fading environment with Additive White Class A Noise (AWCN), which is used to model the actual powerline noise characteristics. The satellite channel for urban area, modeled as a combination of Rayleigh and lognormal processes with the presence of Additive White Gaussian Noise (AWGN) is also studied. System efficiency is enhanced by the application of Orthogonal Frequency Division Multiplexing (OFDM), convolutional coding and block interleaving and the performance of different Phase Shift Keying (PSK) modulation schemes under the imposed channel conditions is investigated. The performance of the system is assessed by the commonly used Bit Error Rate (BER) vs. Signal to Noise Ratio (SNR) diagrams and there is also a comparison regarding the throughput efficiency among the examined systems. As stated in the results section, STBC can be used in powerline and satellite communications providing remarkable results, comparable to those in terrestrial wireless communications where STBC is nowadays a rather mature technique.
\end{abstract}

Index Terms-Space-Time block coding, powerline communications, satellite communications, orthogonal frequency division multiplexing, PSK modulation.

\section{INTRODUCTION}

$\mathbf{T}$ HE idea of using powerlines to provide information as well as power is quite old. Powerline Communications (PLC) offer a potentially convenient and inexpensive solution; the unique fact that no new wires are needed, the availability of power outlets in every room and the simplicity of installation give PLC the opportunity to compete with other "last mile" technologies, such as Digital Subscriber Line (DSL) [1], Wireless Local Loop (WLL), Wireless Local Area Networks (WLANs), etc. Most of the available PLC systems provide a maximum data rate of more than several Mbps [2]. However, the powerline grid can be characterized as a rather hostile medium for data transmission [3] as it was originally designed for the distribution of electrical power in the frequency of $50-60 \mathrm{~Hz}$. As a consequence, the PLC channel faces some technical problems, such as impedance variations and mismatches, various forms of noise (mainly impulsive noise) and narrowband interference, multipath propagation phenomena, high attenuation and other barriers of the medium.

The transmission environment in PLC seems much worse than that in wireless communications. Thus, not only is it

The authors are with the Department of Electrical and Computer Engineering, Aristotle University of Thessaloniki, Panepistimioupolis, 54124 Thessaloniki, Greece. E-mails: \{anpapai, papgeo, niovi\}@auth.gr. essential to utilize existing advanced technologies but also to create novel ones. Several communication techniques have been suggested for application to the powerline channel. The objective for reliable communications is to define a desirable physical layer design that provides low Bit Error Rates (BERs) at low received Signal to Noise Ratio (SNR), performs well in multipath and fading conditions, occupies an affordable bandwidth, and it is easy and cost effective to be implemented. Reed-Solomon codes, repetition coding, block and convolutional coding are different forward error correction techniques that have been proposed for applications recently [4]. Turbo coding is a fairly new coding technique proposed in [5] for improving the performance of PLC systems. Besides, spacetime coding is a coding-modulation technique for multiple emitting and receiving antennas/points [3], which has been investigated recently in PLC [6]-[8], as well as in satellite communications [9]-[11]. It combines temporal and spatial diversity in order to provide less attenuated replicas of the transmitted signal to the receiver and thus to mitigate the destructive effects of attenuation. Careful studies on possible transmission techniques are needed in order to aid the decision procedures for future standards, especially for PLC, where the standardization is still an open process.

Wireless communications are considered to be a more convenient way to provide communication services, as the channel seems to have more favorable propagation characteristics compared to the PLC's. Therefore, the channel is widely used for broadband communications, achieving very low BERs with high data rates, at acceptable SNRs. However, the satellite channel is quite a difficult channel for communications, facing different kinds of noise, attenuation and multipath phenomena. There are several satellite channel models proposed in the literature, out of which Loo's model is investigated in this work as is described in section II.

The outline of this paper is as follows. In section II, the channel models are analyzed. The application of Space-Time Block Coding (STBC) to the channels under study is examined in section III. The system structure and analysis are delineated in section IV. The performance evaluation of the system and extensive simulation results are presented in section $\mathrm{V}$, while conclusion remarks are drawn in section VI.

\section{Channel Models}

\section{A. Powerline channel}

Channel characteristics can be both time- and frequencydependent, and also dependent on the location of the transmitter and the receiver in the specific powerline infrastructure. 
Hence, the channel can be described as random, time varying, having a frequency-dependent SNR over the communication bandwidth. As the powerline grid has been designed for the distribution of energy, the characteristics for data transmission are rather unfavorable: varying impedance, echoes due to the impedance discontinuities, considerable noise and high attenuation. An in-house transfer function, stemming from measurements, shows some deep narrowband notches spread over the whole frequency range. The different noise sources, such as motors, radio signals and power supplies, result in a noise curve very much dependent on frequency, load, time of day and geographical location [3].

In contrast to other communication channels, the powerline channel does not represent an Additive White Gaussian Noise (AWGN) environment [6]. In the frequency range from some hundreds of $\mathrm{kHz}$ up to $20 \mathrm{MHz}$, it is mostly dominated by narrow-band interference and impulsive noise. The impulses have durations from some microseconds up to a few milliseconds with random arrival times. The most suitable model for this type of noise is the Additive White Class A Noise (AWCN).

AWCN is calculated from the combination of AWGN and impulsive noise. The probability density function (pdf) of a Class A noise random (complex) variable $\mathrm{x}$ is given by [7]:

$$
p(x)=\frac{1}{2 \pi} \sum_{m=0}^{\infty} \frac{1}{\sigma_{m}^{2}} \alpha_{m} \exp \left(-\frac{|x|^{2}}{2 \sigma_{m}^{2}}\right)
$$

where: $\alpha_{m}=e^{-A} \frac{A^{m}}{m !}, \sigma_{m}^{2}=\sigma^{2} \frac{(m / A)+T}{1+T}, \sigma^{2}$ is the variance of the Class A noise, $T=\frac{\sigma_{g}^{2}}{\sigma_{t}^{2}}, \sigma_{g}^{2}$ is the variance of the AWGN component and $\sigma_{\iota}^{2}$ is the variance of the impulsive component. The parameter $A$ is called the impulsive index. For small $A$, for example $A=0.1$, the noise is highly impulsive, whereas for $A \rightarrow \infty$ the Class A noise pdf becomes Gaussian.

The samples representing the Class A noise are derived from [12]:

$$
n=x_{G}+\sqrt{K_{m}} y
$$

where $x_{G}$ is a white Gaussian background noise sequence with zero mean and variance $\sigma_{G}^{2}, K_{m}$ is a statistically independent Poisson distributed random sequence whose pdf is characterized by the parameter $A$ (mean value of Poisson distribution), and $y$ is a white Gaussian sequence with zero mean and variance $\sigma_{\iota}^{2} / A$. All random sequences in this model are statistically independent from each other.

Regarding the channel model, there is a lack of a widely accepted PLC model, although several approaches for indoor as well as outdoor networks are proposed in the literature as a result of extensive trials. A significant work in the area of the indoor powerline channel modeling is done by Galli et al. in [13] and [14]. However, this channel model cannot be applied in our work in the form presented in these papers, because the Single-Input Single-Output (SISO) case is considered. This form is not directly applicable to a Multiple-Input MultipleOutput (MIMO) scenario as the one examined in our paper. Such an implementation would demand further study on channel modeling and a different circuit/network topology. Taking into consideration published as well as our own measurements
[1] in order to have a better insight into the variations of the channel, an appropriate model was selected for our study. This is the echo model proposed by Philipps in [15], describing the transmission characteristics in the frequency range of up to $30 \mathrm{MHz}$.

Hence, the powerline channel can be regarded as a multipath environment, where transmitted signals arrive at the receiver not via a direct path, but through $\mathrm{N}$ paths, undergoing different delays and attenuation. The complex attenuation of each path is: $\rho_{\nu}=\left|\rho_{\nu}\right| e^{j \phi_{\nu}}$, with $\phi_{\nu}=\arctan \left(\frac{\operatorname{Im}\left(\rho_{\nu}\right)}{\operatorname{Re}\left(\rho_{\nu}\right)}\right)$, and the N-path propagation model (impulse response) is given by:

$$
h(t)=\sum_{\nu=1}^{N}\left|\rho_{\nu}\right| e^{j \phi_{\nu}} \delta\left(t-\tau_{\nu}\right)
$$

where $N$ is the total number of paths, $\rho_{\nu}$ and $\phi_{\nu}$ are the amplitude and the phase of the attenuation factor respectively, and $\tau_{\nu}$ is the delay of the signal received by the $\nu^{t h}$ path. It can clearly be seen that for each path, a set of only three parameters $\left(\rho_{\nu}, \phi_{\nu}\right.$ and $\left.\tau_{\nu}\right)$ has to be defined. As a consequence, the entire model with $N$ paths is completely defined by $3 N$ parameters. In this study, a 5-path impulse response provided by the Philipps' channel model [15] is taken into consideration. These parameters are presented in table I. As one can observe, the sum of the squares of the five amplitudes $\rho_{\nu}$ is far from approaching unity, which is the aim when as many multipath components as possible are taken into account. This is due to the fact that the powerline channel presents much worse transmission conditions compared to the wireless channel. Thus, each multipath component has very small amplitude and their sum should include an infinite number of components in order to approach unity.

TABLE I: Set of parameters for the powerline echo model

\begin{tabular}{c|c|c|c}
\hline No. of path & $\rho$ & $\varphi$ in $\mathrm{rad}$ & $\tau$ in $\mu \mathrm{s}$ \\
\hline \hline $1^{\text {st }}$ & 0.151 & 0.691 & 0.110 \\
\hline $2^{\text {nd }}$ & 0.047 & -0.359 & 0.154 \\
\hline $3^{\text {rd }}$ & 0.029 & 0.591 & 0.205 \\
\hline $4^{t h}$ & 0.041 & 2.913 & 0.311 \\
\hline $5^{t h}$ & 0.033 & 1.012 & 0.427 \\
\hline
\end{tabular}

\section{B. Satellite channel}

The noise characterizing the satellite channel is AWGN. The so-called Land Mobile Satellite (LMS) channel can be modeled as a sum of three components: two coherent (direct wave and specular wave) and an incoherent one (diffuse wave) [16]. The direct component is received through a line-ofsight (LOS) path. Shadowing is the attenuation of the direct component, caused by any type of obstacles (trees, buildings, hills or mountains). The specular component is generated by signal reflection from the ground, while the diffuse component of the received signal is a multipath wave due to reflections and scattering from the space around the receiver. This component is responsible for the small-scale variations of the signal's amplitude and phase. From the above we can reach the conclusion that shadowing and multipath are the processes that dominate the LMS channel. 
The log-normal distribution is widely used to describe the large-scale fading, which refers to the variations of the signal amplitude caused by the attenuation of the LOS path. The receiver is also surrounded by a large number of scattering objects and multipath propagation mechanism is also an issue to consider. Hence, in urban environments, multipath is well described by the Rayleigh distribution. The resultant distribution, aiming at the description of both small and large-scale fading, is a combination of the above mentioned distributions.

In this work, the LMS channel is modeled using Loo's model, referred to in [16], which is a narrow-band, global statistical (first order statistics) channel model. The so-called specular component of the received signal is ignored and only the direct as well as the diffuse-multipath component are taken into consideration. The direct component suffers from shadowing, while the diffuse component has constant power. The mathematical expression describing the channel process $r$ is:

$$
\mathbf{r}=\mathbf{S} e^{j \phi_{\mathbf{o}}}+\mathbf{R} e^{j \phi}
$$

where the sequence $S$, which corresponds to the direct component (log-normal envelope), is added to the sequence $R$, which represents the diffuse component (Rayleigh envelope), and $\phi_{\mathbf{0}}, \phi$ are uniformly distributed sequences.

\section{Space Time Block Coding}

Space-time coding has comprehensively been studied in terrestrial wireless channels with very good performance results. The fundamental phenomenon that makes reliable wireless transmission a hard task is time-varying multipath fading [17]. In most cases, the wireless channel suffers from attenuation due to the destructive addition of multipaths in the propagation media and interference from other users [18].

Reducing the error rate in a multipath fading environment is not an easy task. Theoretically, the most effective technique to mitigate multipath fading in a wireless channel [17], as well as in PLC, is transmitter power control. However, the improvement in SNR may not be achieved by higher transmit power (causing Electromagnetic Compatibility -EMC- problems) or additional bandwidth.

Another effective technique is diversity, which can be provided using temporal, frequency, polarization and spatial resources [18]. In most scattering environments, antenna diversity is a practical and widely applied method aiming at ameliorating the detrimental effects of multipath fading. Space-time coding implements the idea of using multiple transmitting and multiple receiving antennas by the linear combination of the transmitted signal at the transmitter, while the inverse process takes place at the receiver [17].

The application of STBC to the powerline channel presents some basic differences in comparison with the wireless channel. In wireless communications multiple transmitting and receiving antennas can be used. In the PLC environment, where the implementation of space-time codes can take advantage of the intrinsic spatial diversity in the use of a threephase powerline network [19], there exist two cases: the first scenario considers three emitting and three receiving points [6], which are the three phases of the powerline channel, and can be applied to the "last mile" environment. The second one, proposed in this paper, implements two emitting and two receiving points assuming communication via two channels, phase - protective earth and neutral - protective earth and can be applied to an in-home network (LAN). This decision was taken under the consideration of [20], where the SISO case is described. Besides, in wireless communications with multiple transmitting and receiving antennas, the signal at the input of each receiving antenna is calculated through the linear combination of the transmitted signals of all the transmitting antennas. On the other hand, in PLC, the wires are assumed to be completely isolated [6]. This consideration is an assumption made in this work, which simplifies the signal transmission through the powerline channel [13]. However, in the actual powerline network there is energy transfer between the wires, due to wiring and grounding practices, which can lead to diversity gain at the receiver. In the wireless case the diversity order is $M \cdot N$ for $M$ transmitting and $N$ receiving antennas.

Space-Time Block Codes are defined by the transmission matrix G. In our system the general structure of the transmission matrix, employing two transmitting and two receiving points for complex orthogonal design [21], is:

$$
\mathbf{G}_{c}^{2}=\left[\begin{array}{cc}
x_{1} & x_{2} \\
-x_{2}^{*} & x_{1}^{*}
\end{array}\right]
$$

where the $i$-th row represents the symbols transmitted during the $i$-th time slot and the $j$-th column the symbols transmitted from the $j$-th emitting point. Generally, the code rate is defined as $R=k / p$, since $p$ time slots are used to transmit $k$ symbols [18]. For complex constellations, the transmission matrix with dimensions $2 \times 2$ is the only one that can achieve rate 1 [7], which is also the case investigated in this work.

The inverse process takes place at the receiver, where the space-time decoding is performed. Assuming two transmitting $\left(T x_{1}, T x_{2}\right)$ and two receiving antennas $\left(R x_{1}, R x_{2}\right)$ (figure 1 case $b)$, the received signals are:

$$
\begin{aligned}
& r_{0}=r_{1}(t)=h_{0} x_{1}+h_{1} x_{2}+n_{0} \\
& r_{1}=r_{1}(t+T)=-h_{0} x_{2}^{*}+h_{1} x_{1}^{*}+n_{1} \\
& r_{2}=r_{2}(t)=h_{2} x_{1}+h_{3} x_{2}+n_{2} \\
& r_{3}=r_{2}(t+T)=-h_{2} x_{2}^{*}+h_{3} x_{1}^{*}+n_{3}
\end{aligned}
$$

where $h_{0}, h_{1}, h_{2}, h_{3}$ are the channel gains between $T x_{1}$ and $R x_{1}, T x_{2}$ and $R x_{1}, T x_{1}$ and $R x_{2}, T x_{2}$ and $R x_{2}$ respectively, and $n_{0}, n_{1}, n_{2}, n_{3}$ are the noise and the interference samples.

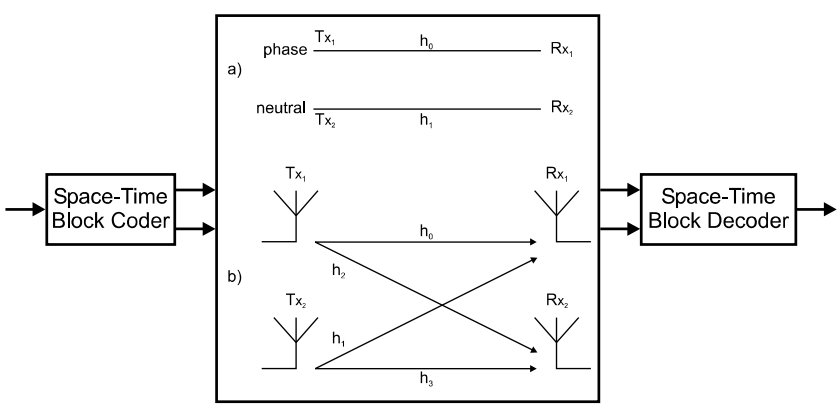

Fig. 1: Space-Time Block Coding Transmission 
The combiner constructs the symbols $x_{1}^{\prime}$ and $x_{2}^{\prime}$ given below, which are then sent to the detector.

$$
\begin{aligned}
& x_{1}^{\prime}=h_{0}^{*} r_{0}+h_{1} r_{1}^{*}+h_{2}^{*} r_{2}+h_{3} r_{3}^{*} \\
& x_{2}^{\prime}=h_{1}^{*} r_{0}-h_{0} r_{1}^{*}+h_{3}^{*} r_{2}-h_{2} r_{3}^{*}
\end{aligned}
$$

For Phase Shift Keying (PSK) signals [17], the maximum likelihood (ML) detector computes the square of the Euclidean distance between $x_{1}^{\prime}$ and each constellation symbol $x_{k}$, and decides in favor of $x_{i}$ if:

$$
d^{2}\left(x_{1}^{\prime}, x_{i}\right) \leq d^{2}\left(x_{1}^{\prime}, x_{k}\right), \quad \forall i \neq k
$$

For the powerline channel with two emitting and two receiving points (figure 1 , case a), perfect isolation between the wires is assumed. Thus, equations (6) are simplified:

$$
\begin{aligned}
& r_{0}=r_{1}(t)=h_{0} x_{1}+n_{0} \\
& r_{1}=r_{1}(t+T)=-h_{0} x_{2}^{*}+n_{1} \\
& r_{2}=r_{2}(t)=h_{1} x_{2}+n_{2} \\
& r_{3}=r_{2}(t+T)=h_{1} x_{1}^{*}+n_{3}
\end{aligned}
$$

The ML detector receives the combined signals:

$$
\begin{aligned}
& x_{1}^{\prime}=h_{0}^{*} r_{0}+h_{1} r_{3}^{*} \\
& x_{2}^{\prime}=-h_{0} r_{1}^{*}+h_{1}^{*} r_{2}
\end{aligned}
$$

and uses the decision criterion of (8).

Furthermore, the channel capacity is examined. For a SISO system, the capacity expressed in bits/sec/Hz is given by:

$$
C=\log _{2}\left(1+\rho|h|^{2}\right)
$$

where $h$ is the normalized complex gain of a particular realization of a random channel and $\rho$ is the SNR at any transmitting antenna [22].

The channel capacity for a MIMO system with $N$ transmitting and $M$ receiving antennas is computed using the equation from [23]:

$$
C=\log _{2}\left[\operatorname{det}\left(\mathbf{I}_{M}+\frac{\rho}{N} \mathbf{H H}^{*}\right)\right]
$$

where $\mathbf{H}$ is the $M \times N$ channel matrix and $\left(^{*}\right)$ denotes the transpose-conjugate.

When STBC is used, the channel capacity is given by [24]:

$$
C=R \log _{2}\left(1+\frac{1}{N R} S N R \sum_{m=1}^{M} \sum_{n=1}^{N}\left|h_{n, m}\right|^{2}\right)
$$

where $S N R$ is the total mean signal to noise power ratio at each receiving antenna, $R$ is the space-time code rate and $h_{n, m}$ is the channel gain from the transmitting antenna $n$ to the receiving antenna $m$. Equation (13) can be written equivalently:

$$
C=R \log _{2}\left(1+\frac{1}{N R} S N R\|\mathbf{H}\|_{F}^{2}\right)
$$

where $\|\mathbf{H}\|_{F}^{2}$ is the squared Frobenius norm of channel matrix H.

The use of the Shannon's standard capacity formula $C=$ $B W \log _{2}(1+S N R)$ is not directly applicable to powerline channels, since the $S N R$ is not constant within the bandwidth
$B W$ [25]. Thus, the capacity is computed taking into account the received signal power spectral density (PSD) $\Phi_{r}(f)$ and a frequency dependent noise PSD $\Phi_{n}(f)$ :

$$
C=\int_{f_{L}}^{f_{U}} \log _{2}\left(1+\frac{\Phi_{r}(f)}{\Phi_{n}(f)}\right) d f, \text { with } B W=f_{U}-f_{L}
$$

where $\Phi_{r}(f)=\Phi_{t}(f) \cdot|H(f)|^{2}, \Phi_{t}(f)$ is the transmission PSD and $H(f)$ is the channel transfer function. Considering the SNR at the transmitter $\frac{\Phi_{t}(f)}{\Phi_{n}(f)}$ in $\mathrm{dB}$, the powerline channel capacity can be computed by equation (15) in the frequency range of 1-30 MHz.

\section{System Structure AND ANALYsis}

The general layout of the simulated system can be seen in figure 2. The data to be transmitted is first coded with convolutional coding $(2,1)$ with generator polynomial $[133,171]$. The reason for utilizing convolutional coding is that it is widely used in the literature with very good performance results. However, in fading channels with high levels of impulsive noise, like the powerline channel, errors have a bursty nature. This can be controlled by using burst error correcting techniques, i.e. block interleaving [26]. In this work the signal is block interleaved with interleaving depth 40 .

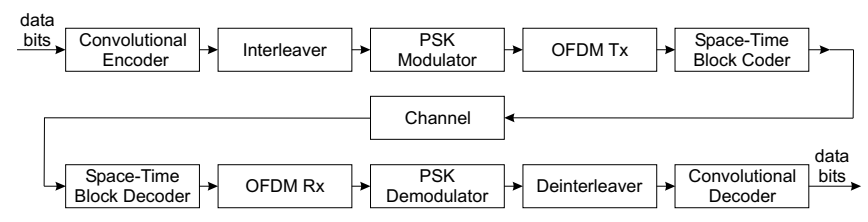

Fig. 2: System Structure

The modulation schemes under examination are the commonly used Binary Phase Shift Keying (BPSK) and Quadrature Phase Shift Keying (QPSK), which are the most popular in applications to wireless and powerline channels today. The signal power for both modulation schemes is normalized to unity.

Orthogonal Frequency Division Multiplexing (OFDM) is a multicarrier modulation scheme suitable for PLC due to its ability to deal with multipath propagation, intersymbol interference and frequency selective channels. In OFDM the signal is modulated through 512 carriers, equally spaced within the frequency range from 1 to $30 \mathrm{MHz}$. The guard time in the case of the satellite channel is a quarter of the OFDM symbol duration, while for the powerline channel the guard time is chosen to be $0.5 \mathrm{sec}$, greater than the maximum delay spread $(0.427 \mathrm{sec})$.

The STBC scheme applied in our study utilizes two emitting points / antennas and two receiving points/ antennas for the examined channels (hereafter referred to as STBC2x2). The transmission matrix used to produce the space-time encoder and decoder was described in (5). For the PLC application it is essential to use equal number of transmitting and receiving points. On the contrary, implementation in wireless channels employs an arbitrary number of antennas. However, STBC2x2 is studied for both the powerline and the satellite environment. 
The Class A noise is characterized by three parameters [27], which were mentioned in section II-A. These are the impulsive index $A$, the variance of the AWGN component $\sigma_{g}^{2}$ and the ratio $T$. Based on [27], $A$ is set equal to 0.25 , $\sigma_{g}^{2}=10^{-3} W$ and $T=0.001$. The parameter $A$ determines the "impulsiveness" of Class A noise (i.e. it is the product of the mean number of impulses per second and the mean length of an impulse in seconds). Comparing with other impulsive noise scenarios studied in [28], it can be concluded that choosing $A$ to be 0.25 represents very heavy noise conditions.

In the following section, simulation results for the terrestrial wireless channel are also presented, for the sake of comparison with the benefits that STBC can offer to powerline and satellite channels. For the terrestrial wireless channel, the channel gains are modeled as samples of independent complex Gaussian random variables with variance 0.5 per real dimension, which results in a Rayleigh distribution with variance 1 [7]. The path gains used for the simulation of the powerline channel are given in table I. For the satellite channel, the channel gains are given by the sum of a log-normal and a Rayleigh distribution as described in section II-B. It should be mentioned here that perfect channel estimation at the receiver is assumed in all cases.

\section{Performance Evaluation And Simulation RESULTS}

For the performance evaluation of the proposed systems, a simulation was developed in Matlab. The figures presented at the end of the paper were obtained by averaging the results of multiple simulation runs, in order to minimize the statistical errors and to assure the validity of the results. The system was evaluated by simulating a transmission of about $2^{20}$ (1048576) data bits, so as to derive the performance results for each one of the tested systems. The multiparametric nature of the simulation should be mentioned, including the following: bits per symbol, modulation techniques and interleaving depth. These are the major parameters with which we tested the system performance for several values and combinations, in order to decide on the optimal ones. In this section, the BER

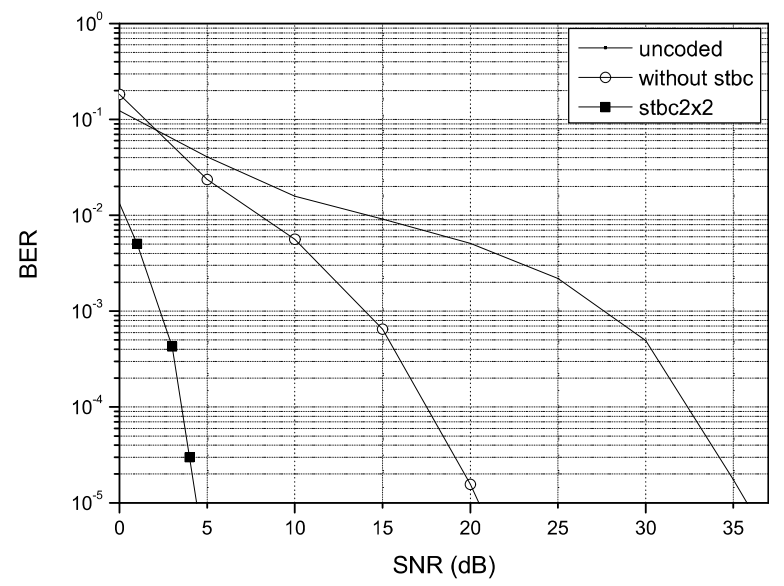

Fig. 3: BER performance for the PLC channel using BPSK modulation

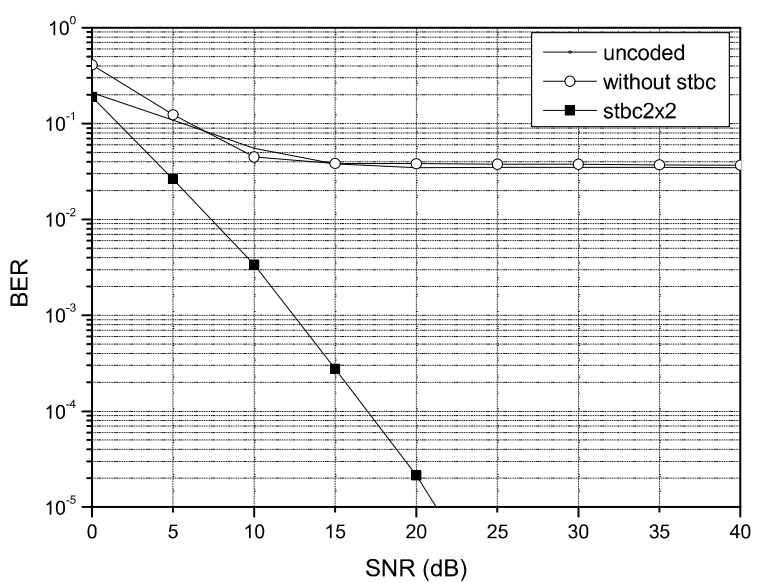

Fig. 4: BER performance for the PLC channel using QPSK modulation

and the bitrate performance of the aforementioned systems are presented for a wide range of SNRs.

In figures 3 and 4 the PLC channel is examined using BPSK and QPSK modulations respectively. In the first case, it is apparent that even a simple convolutional code combined with interleaving yields much better results compared to the uncoded transmission. When STBC is applied the system performs even better, with STBC inducing a gain of about 16 $\mathrm{dB}$ at a BER of $10^{-5}$. In the case that the signal is modulated with QPSK, the results of the simulation show that low BER can be achieved only by the implementation of space-time coding, otherwise increasing the SNR does not improve the performance. Based on [28], this error floor can be justified as a result of the very heavy impulsive noise scenario used in our work. The overall results are still worse than those for BPSK, as expected.

The results for the satellite channel are presented in figures 5 and 6. In the case that BPSK is implemented (figure 5), the scheme without STBC reaches a BER of $10^{-5}$ at an SNR of $32.5 \mathrm{~dB}$, while the STBC2x2 attains the same BER at about $14 \mathrm{~dB}$. Regarding the QPSK system in figure 6, a

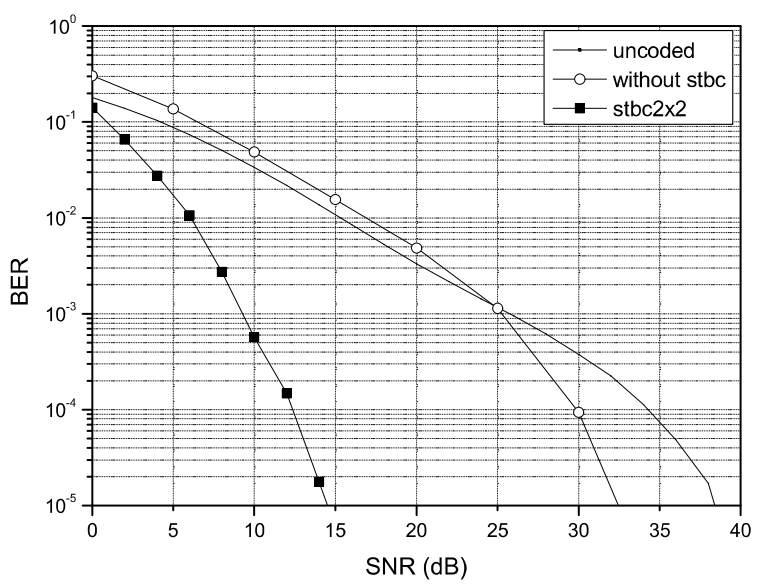

Fig. 5: BER performance for the satellite channel using BPSK modulation 


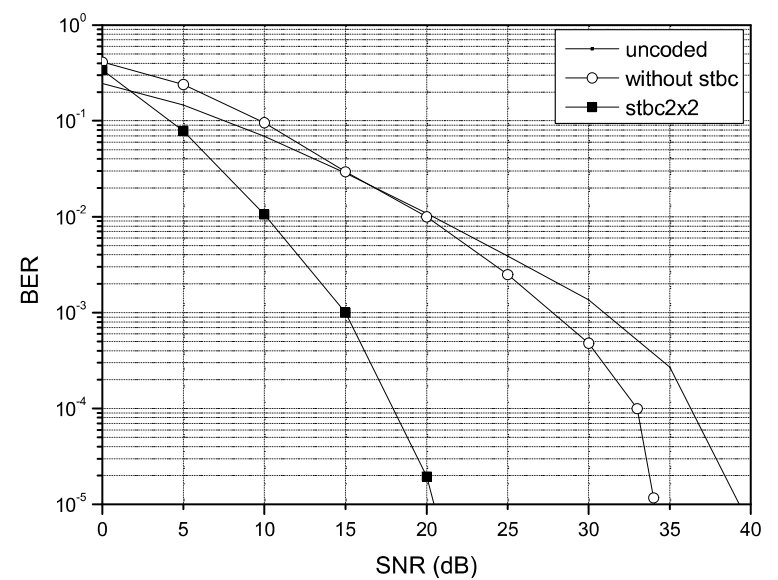

Fig. 6: BER performance for the satellite channel using QPSK modulation

deterioration to the previous figure's curves can be observed when comparing the two modulation schemes. It can be stated here that STBC2x2 offers less gain to the non-STBC system (12.5 dB at a BER of $\left.10^{-5}\right)$ than that in the BPSK case.

Results for the terrestrial wireless channel are presented in figures 7 and 8 as a reference for comparison with powerline and satellite channels. In figure 7, where BPSK modulation is applied, STBC is quite beneficial, as it can reduce the required SNR by more than $16 \mathrm{~dB}$ at a BER of $10^{-5}$ compared to the system without STBC. Figure 8 depicts the efficiency of the QPSK modulation. The system performance shows a similar behaviour as with BPSK, with STBC2x2 providing a gain of $16 \mathrm{~dB}$ at a BER of $10^{-5}$ compared to the case without STBC.

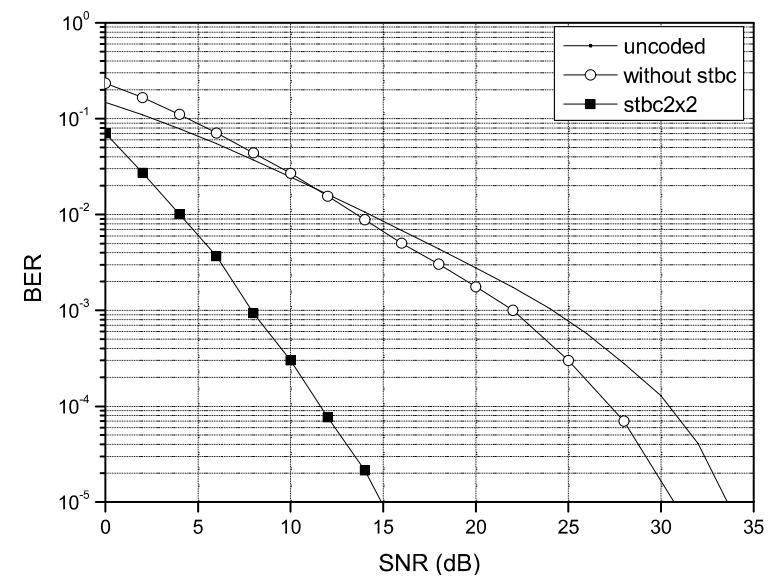

Fig. 7: BER performance for the terrestrial wireless channel using BPSK modulation

As a general comment, it can be seen that STBC is profitable not only for terrestrial wireless communications, but for PLC and satellite communications as well. Regarding the PLC channel, STBC seems more beneficial when the signal is modulated with QPSK than with BPSK. It can be observed in all figures that the utilization of $\mathrm{STBC} 2 \times 2$ offers a great improvement compared to the non-STBC system.

The capacity of systems under study is presented in figures

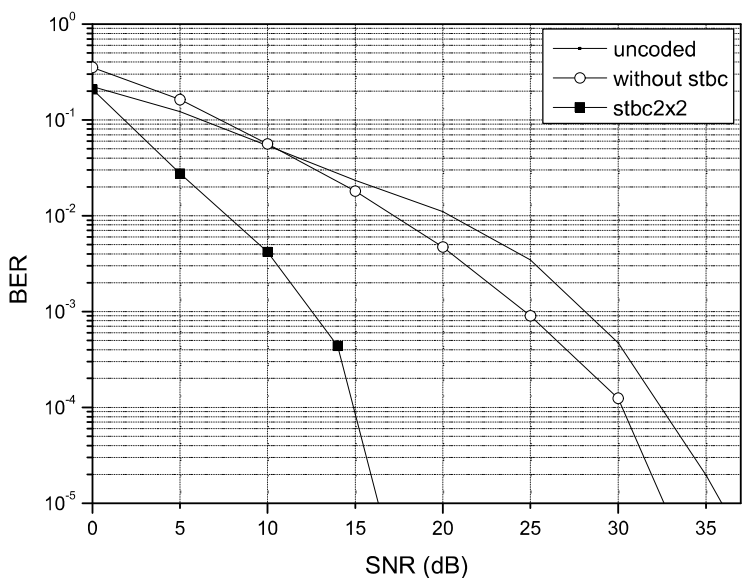

Fig. 8: BER performance for the terrestrial wireless channel using QPSK modulation

9 - 11. For the powerline channel, the capacity with respect to mean receiver SNR is computed in the frequency range of 1-30 MHz for both the SISO and the STBC2x2 cases (figure 9). As it can be noticed, there is almost no difference between the SISO and the STBC2x2 capacities. This happens due to the considered assumption (isolation of the two wires). If we

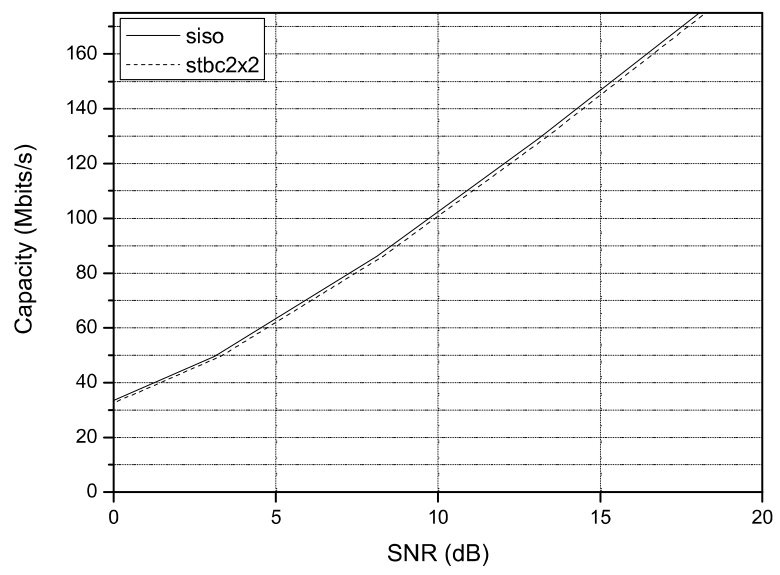

Fig. 9: Capacity for powerline channel

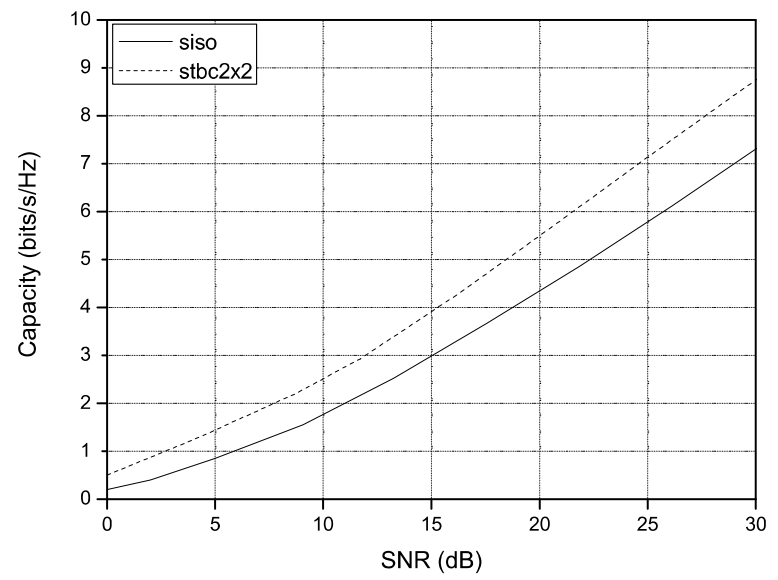

Fig. 10: Ergodic capacity for satellite channel 


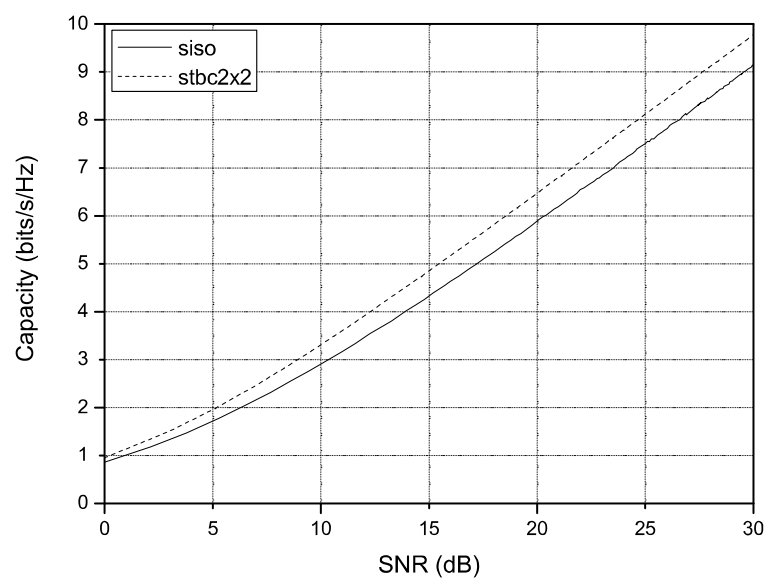

Fig. 11: Ergodic capacity for terrestrial wireless channel

had not taken into account this assumption, better behaviour of the capacity would be expected when using STBC, because the diversity gain would increase. On the contrary, regarding the ergodic (mean) capacity comparison in figures 10 and 11 , it can be seen that STBC2x2 offers higher capacity to the system than the SISO case. It is known that a full rate STBC, used over any channel with one receive antenna, is always optimal with respect to capacity [22] and that capacity increases with increasing number of antennas, due to diversity order. The improvement in capacity is more evident for the satellite channel compared to the wireless channel. The amelioration that STBC provides to the capacity of the wireless systems is obvious, in contrast to the powerline system.

\section{CONCLUSIONS}

This paper examines the BER performance as well as the channel capacity of an STBC system, for both powerline and satellite channels. BPSK and QPSK modulations combined with convolutional code are also investigated. In addition, OFDM is implemented in order to reinforce system's robustness against multipath propagation phenomena and interference. Interleaving is also evaluated as means to improve this performance.

The STBC application appears to be quite beneficial in terms of the BER performance for both the PLC and the LMS channel. STBC seems to provide a gain of more than $10 \mathrm{~dB}$ at a BER of $10^{-5}$ in comparison with the non-STBC system, for the two modulation schemes and the different communication environments. The capacity of the wireless simulated systems seems to increase when STBC of rate one is applied, while for PLC it remains the same. The overall conclusion coming out of this study is that both powerline and satellite communications can profit a lot from the implementation of this codingmodulation technique.

In conclusion, it should be pointed out that there is a lot of further research to be done, investigating the application of STBC in the powerline and satellite communications. For future work towards this direction we intend to study how the efficiency of STBC is affected, examining in depth the MIMO powerline channel modeling and the effect of the noise correlation in the powerline network.

\section{REFERENCES}

[1] C. Assimakopoulos, P. L. Katsis, F.-N. Pavlidou, D. Obradovic, and M. Obradovic, "xDSL Techniques for Power Line Communications," in Proc. of International Symposium on Power-Line Communications and Its Applications 2003 (ISPLC 2003), Kyoto, Japan, Mar. 2003, pp. 21-25.

[2] W. Liu, H. Widmer, and P. Raffin, "Broadband PLC Access Systems and Field Deployment in European Power Line Networks," IEEE Commun. Mag., vol. 41, no. 5, pp. 114-118, May 2003.

[3] F.-N. Pavlidou, A. J. H. Vinck, and J. Yazdani, "Power Line Communications: State of the Art and Future Trends," IEEE Commun. Mag., vol. 41, no. 4, pp. 34-40, Apr. 2003.

[4] P. L. Katsis, G. D. Papadopoulos, and F.-N. Pavlidou, "Coded MCCDMA Systems for Power Line Communications," in TELSIKS 2003, Nis, Serbia and Montenegro, Oct. 2003, pp. 153-156.

[5] P. Katsis, G. Papadopoulos, and F.-N. Pavlidou, "Comparison of Coded Orthogonal Frequency Division Multiplexing and Multicarrier Code Division Multiple Access Systems for Power Line Communications," International Journal of Communication Systems (IJCS), vol. 17, no. 9, pp. 833-850, Nov. 2004.

[6] C. L. Giovaneli, P. Farrell, and B. Honary, "Application of SpaceTime Block Codes for Power Line Communication Channels," in Proc. of Communication Systems, Networks and Digital Signal Processing Symposium, Staffordshire, U.K., July 2002.

[7] C. L. Giovaneli, J. Yazdani, P. Farrell, and B. Honary, "Application of Space-Time Diversity/Coding for Power Line Channels," in Proc. of International Symposium on Power-Line Communications and Its Applications 2002 (ISPLC 2002), Athens, Greece, Mar. 2002, pp. 101105.

[8] A. Papaioannou, G. D. Papadopoulos, and F.-N. Pavlidou, "Performance of Space-Time Block Coding over the Power Line Channel in Comparison with the Wireless Channel," in Proc. of International Symposium on Power-Line Communications and Its Applications 2004 (ISPLC 2004), Saragossa, Spain, Apr. 2004, pp. 362-366.

[9] M. Uysal and C. N. Georghiades, "Effect of Shadowing on the Performance of Space-Time Trellis-Coded Systems," IEEE Trans. Wireless Commun., vol. 3, no. 4, pp. 1037-1042, July 2004.

[10] M. Uysal, "Pairwise Error Probability of Space-Time Codes in RicianNakagami Channels," IEEE Commun. Lett., vol. 8, no. 3, pp. 132-134, Mar. 2004.

[11] R. Tesi, D. Tujkovic, E. Kunnari, and L. Mucchi, "Space-Time Coded Satellite Diversity in S-UMTS," in 7th International Workshop on Digital Signal Processing Techniques for Space Communications, Sesimbra, Portugal, Oct. 2001.

[12] G. Pay and M. Safak, "Performance of DMT Systems under Impulsive Noise," in Proc. of International Symposium on Power-Line Communications and Its Applications 2001 (ISPLC 2001), Malmo, Sweden, Apr. 2001, pp. 109-114.

[13] S. Galli and T. C. Banwell, "Modeling the Indoor Power Line Channel: New Results and Modem Design Considerations," in Proc. of IEEE Consumer Communications and Networking Conference (CCNC'04), Las Vegas, USA, Jan. 2004.

[14] S. Galli and T. Banwell, "A Novel Approach to the Modeling of the Indoor Power Line Channel - Part II: Transfer Function and Its Properties," IEEE Trans. Power Delivery, vol. 20, no. 3, pp. 1869-1878, July 2005

[15] H. Philipps, "Modelling of Powerline Communication Channels," in Proc. of International Symposium on Power-Line Communications and Its Applications 1999 (ISPLC 1999), Lancaster, UK, Mar. 1999, pp. 1421.

[16] M. Karaliopoulos and F.-N. Pavlidou, "A Review on Modeling of the Land Mobile Satellite Channel," IEE Electronics and Communication Engineering Journal, vol. 11, no. 5, pp. 235-248, Oct. 1999.

[17] S. M. Alamouti, "A Simple Transmit Diversity Technique for Wireless Communications," IEEE J. Select. Areas Commun., vol. 16, no. 8, pp. $1451-1458$, Oct. 1998.

[18] V. Tarokh, H. Jafarkhami, and A. R. Calderbank, "Space-Time Block Coding for Wireless Communications: Performance Results," IEEE J. Select. Areas Commun., vol. 17, no. 3, pp. 451-460, Mar. 1999.

[19] E. Biglieri, "Coding and Modulation for a Horrible Channel," IEEE Commun. Mag., vol. 41, no. 5, pp. 92-98, May 2003.

[20] K. Dostert, Powerline Communications. New Jersey: Prentice Hall, 2001.

[21] V. Tarokh, H. Jafarkhami, and A. R. Calderbank, "Space-Time Block Codes from Orthogonal Designs," IEEE Trans. Inform. Theory, vol. 45, no. 5, pp. 1456-1467, July 1999. 
[22] S. Sandhu and A. Paulraj, "Space-Time Block Codes: A Capacity Perspective," IEEE Commun. Lett., vol. 4, no. 12, pp. 384-386, Dec. 2000.

[23] D. Gesbert, M. Shafi, D.-S. Shiu, P. J. Smith, and A. Naguib, "From Theory to Practice: An Overview of MIMO Space-Time Coded Wireless Systems," IEEE J. Select. Areas Commun., vol. 21, no. 3, pp. 281-302, Apr. 2003.

[24] H. C. Espinosa, J. R. Fonollosa, and J. A. D. Penin, "Channel Capacity of Space-Time Block Coding," in IST Mobile Communications Summit 2001, Sitges (Barcelona), Spain, Sept. 2001.

[25] K. Dostert, "New PLC Approaches for High Speed Indoor Digital Networks," in Proc. of International Symposium on Power-Line Communications and Its Applications 2001 (ISPLC 2001), Malmo, Sweden, Apr. 2001, pp. 253-258.

[26] J. G. Proakis and M. Salehi, Communication System Engineering. New Jersey: Prentice Hall, 1994

[27] L. A. Berry, "Understanding Middleton's Canonical Formula for Class A Noise," IEEE Trans. Electromagn. Compat., vol. 23, no. 4, pp. 337-344, Nov. 1981

[28] Y. H. Ma, P. L. So, E. Gunawan, and Y. L. Guan, "Modeling and Analysis of the Effect of Impulsive Noise on Broadband PLC Networks," in Proc. of International Symposium on Power-Line Communications and Its Applications 2004 (ISPLC 2004), Saragossa, Spain, Apr. 2004, pp. $45-50$.

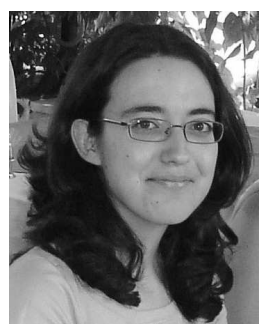

Anna Papaioannou received the Diploma in Electrical and Computer Engineering from the Aristotle University of Thessaloniki, Greece in 2003. She is currently a $\mathrm{PhD}$ candidate at the same department. Her research interests include coding and modulation techniques for powerline communications, space-time coding, cooperative diversity and phymac cross-layer design. She is a student member of the IEEE and a member of the Technical Chamber of Greece.

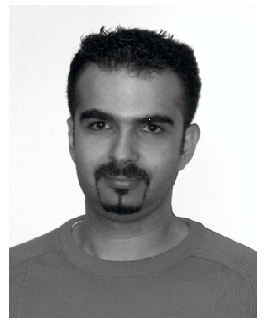

George D. Papadopoulos received his Diploma in Electrical and Computer Engineering from the Aristotle University of Thessaloniki, Greece in 2002. $\mathrm{He}$ is currently a Ph.D candidate in the same department. His research interests include coding techniques and multicarrier systems in multipath and fading channels, such as wireless and powerline communications. He has been involved in many research European and national projects and he has served as a reviewer for many IEEE/IEE journals and conferences. $\mathrm{He}$ is teaching in the aforementioned department. $\mathrm{He}$ is a student member of the IEEE and a member of the Technical Chamber of Greece.

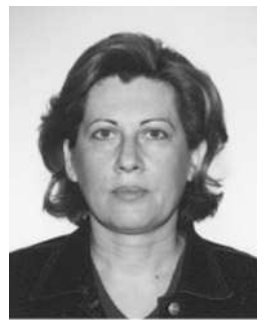

Fotini-Niovi Pavlidou received the PhD degree and the Diploma in Electrical Engineering from the Aristotle University of Thessaloniki (AUTh), Greece, in 1988 and in 1979 respectively. She is with the Dept. of Electrical and Computer Engineering at AUTh engaged in teaching in the areas of Mobile communications and Telecommunications Networks. Her research interests are in the field of mobile and personal communications, satellite communications, multiple access systems, routing and traffic flow in networks and QoS studies for multimedia applications over the Internet. She is participating in many national and international projects (Tempus, COST, Telematics, IST) and she has been chairing the European COST262 Action on "Spread Spectrum Systems and Techniques for Wired and wireless Communications". She has served as member of the TPC in many IEEE/IEE conferences and she has organized/chaired some conferences like, the "IST Mobile Summit2002", the 6th "International Symposium on Power Lines Communications-ISPLC2002", the "International Conference on Communications-ICT1998" etc. She is a permanent reviewer for many IEEE/IEE journals. She has published more than 100 papers in refereed journals and conferences. She has served as guest-editor for special issues in many journals. She is a senior member of IEEE, currently chairing the joint IEEE VT\&AES Chapter in Greece. 